\title{
Altered cutaneous immune parameters in transgenic mice overexpressing viral IL-10 in the epidermis
}

\author{
Wanhong Ding, ${ }^{1}$ Stefan Beissert,${ }^{2}$ Liang Deng, ${ }^{1}$ Edward Miranda, ${ }^{1}$ Christopher Cassetty, ${ }^{1}$ \\ Kristina Seiffert, ${ }^{1}$ Kristina L. Campton, ${ }^{1}$ Zhengmin Yan, ${ }^{3}$ George F. Murphy, ${ }^{4}$ \\ Jeffrey A. Bluestone, ${ }^{5}$ and Richard D. Granstein ${ }^{1}$
}
${ }^{1}$ Department of Dermatology, Joan and Sanford I. Weill Medical College of Cornell University, New York, New York, USA ${ }^{2}$ Department of Dermatology, University of Muenster, Muenster, Germany
${ }^{3}$ Department of Radiology, State University of New York, Stony Brook University Hospital and Health Sciences Center, Stony Brook, New York, USA
${ }^{4}$ Department of Pathology, Jefferson Medical College, Philadelphia, Pennsylvania, USA
${ }^{5}$ Department of Medicine, University of California-San Francisco, San Francisco, California, USA

\begin{abstract}
IL-10 is a pleiotropic cytokine that inhibits several immune parameters, including Th1 cell-mediated immune responses, antigen presentation, and antigen-specific $T$ cell proliferation. Recent data implicate IL-10 as a mediator of suppression of cell-mediated immunity induced by exposure to UVB radiation $(280-320 \mathrm{~nm})$. To investigate the effects of IL-10 on the cutaneous immune system, we engineered transgenic mice that overexpress viral IL-10 (vIL-10) in the epidermis. vIL-10 transgenic mice demonstrated a reduced number of $\mathrm{I}_{-} \mathrm{A}^{+}$epidermal and dermal cells and fewer $\mathrm{I}-\mathrm{A}^{+}$hapten-bearing cells in regional lymph nodes after hapten painting of the skin. Reduced CD80 and CD86 expression by I-A ${ }^{+}$ epidermal cells was also observed. vIL-10 transgenic mice demonstrated a smaller delayed-type hypersensitivity response to allogeneic cells upon challenge but had normal contact hypersensitivity to an epicutaneously applied hapten. Fresh epidermal cells from vIL-10 transgenic mice showed a decreased ability to stimulate allogeneic $\mathrm{T}$ cell proliferation, as did splenocytes. Additionally, chronic exposure of mice to UVB radiation led to the development of fewer skin tumors in vIL-10 mice than in WT controls, and vIL-10 transgenic mice had increased splenic NK cell activity against YAC-1targets. These findings support the concept that IL-10 is an important regulator of cutaneous immune function.
\end{abstract}

J. Clin. Invest. 111:1923-1931 (2003). doi:10.1172/JCI200315722.

\section{Introduction}

The effect of IL-10 on modulation of the cutaneous immune system has been studied in several systems, including delayed-type hypersensitivity (DTH), contact hypersensitivity (CHS), and allograft transplantation. IL-10 negatively regulates a variety of immune responses $(1,2)$ and acts, at least in part, by altering APC function. IL-10 was originally identified as a Th2 cell product that inhibited Th1 cell clones by downregulating IFN- $\gamma$ and IL-2 expression (3). It also downregulates MHC class II antigen expression by monocytes and inhibits antigen presentation by several types of APC, including epidermal Langerhans cells (LCs) $(4,5)$. IL-10 also inhibits the

Received for publication April 17, 2002, and accepted in revised form April 22, 2003.

Address correspondence to: Richard D. Granstein, Department of Dermatology, Weill Medical College of Cornell University, 1300 York Avenue, Room F-342, New York, New York 10021, USA. Phone: (212) 746-7274; Fax: (212) 746-8656;

E-mail: rdgranst@med.cornell.edu.

Wanhong Ding and Stefan Beissert contributed equally to this work.

Conflict of interest: The authors have declared that no conflict of interest exists.

Nonstandard abbreviations used: delayed-type hypersensitivity (DTH); contact hypersensitivity (CHS); Langerhans cell (LC); viral IL-10 (vIL-10); epidermal cell (EC); phycoerythrin (PE);

dinitrofluorobenzene (DNFB); lactic dehydrogenase (LDH). production of cytokines such as IL-1, IL-6, IL-8, TNF- $\alpha$, GM-CSF, G-CSF, and IL-12 (6-9). IL-10 has growth factor activity for several cell types, including thymocytes, some T cells, mast cells, and B cells (10). The capacity of IL-10 to suppress the efferent phase of DTH and CHS supports an important role for this cytokine in the regulation of type IV immune reactions $(3,11)$.

Viral IL-10 (vIL-10), a product encoded by EBV (human herpes virus 4), is highly homologous to both murine and human IL-10 in CDNA sequence. The human IL-10 and vIL-10 mature-protein sequences are $84 \%$ identical, with most of their divergence found in the $\mathrm{NH}_{2}$-terminal 20 amino acids (12-14), especially in the coding region of the mature protein (13). The protein product of vIL-10 shares many biologic properties with both murine and human IL-10 (14). It has immunosuppressive effects that are mediated by inhibition of cytokine synthesis (IL-2 and IFN- $\gamma$ ) by human PBMCs $(1,7,15)$, and it strongly reduces antigen-specific human $\mathrm{T}$ cell proliferation by diminishing the antigen-presenting capacity of monocytes via downregulation of MHC class II expression (6). However, vIL-10 does not possess the T cell costimulatory activities of mammalian cellular IL-10, and it induces increased expression of class II MHC molecules on murine $B$ cells $(6,16,17)$. vIL-10 also inhibits collageninduced arthritis in mice $(18,19)$. These results suggest 
that vIL-10 could be a useful cytokine for blunting the immune response, potentially in the setting of cutaneous autoimmune disease.

To examine the effects of increased IL-10 production within the skin, we generated transgenic mice that overexpress vIL-10 in the epidermis.

\section{Methods}

Construction of transgenic mice. We produced transgenic mice that express vIL-10 in the basal layer of the epidermis, by using the K14/human growth hormone (hgH) expression vector to produce a K14/vIL-10 construct. A $600-\mathrm{bp}$ vIL-10 cDNA was cloned into the unique BamHI site of the $\mathrm{K} 14 / \mathrm{hgH}$ expression vector. Transgenic mice were produced by standard methods at the Brigham and Women's Hospital Core Transgenic Mouse Facility (Boston, Massachusetts, USA). The construct was injected into pronuclei of fertilized eggs from WT female FVB mice to generate vIL-10 transgenic mice (vIL-10Tg). To screen the offspring for the presence of the transgene, PCR typing of mouse tail DNA using a set of K14/IL-10 primer (3'-TTGACGTATCTTCGTATGTACTAC-5', 5'-CATCACCCACAGGCTAGCGCCAAC- $3^{\prime}$ ) was performed. Positive offspring were selected and bred. Homozygous mice were selected through mating between heterozygous siblings. Southern blotting analysis was used to distinguish homozygous from heterozygous or WT animals. All experiments were performed with vIL-10Tg FVB mice and control WT FVB mice.

RT-PCR. Epidermal cells (ECs) were prepared from WT FVB mice and vIL-10Tg mice using a standard protocol (20). Briefly, truncal skins of shaved and chemically depilated mice (Neet; Whitehall Laboratories, New York, New York, USA) were obtained, and subcutaneous fat and panniculus carnosus were removed by blunt dissection. The skins were floated dermis-side-down on 0.5 $\mathrm{U} / \mathrm{ml}$ dispase and $0.4 \%$ trypsin in PBS for 50 minutes at $37^{\circ} \mathrm{C}$. Epidermal sheets were collected and dissociated by gentle stirring for 20 minutes. The resulting ECs were filtered through a 40- $\mu \mathrm{m}$ nylon cell strainer (Becton Dickinson Labware, Franklin Lakes, New Jersey, USA). Total cellular RNA was isolated from ECs using a total RNA extraction kit (QIAGEN Inc., Valencia, California, USA). To remove any contaminating DNA, RNA was treated with RNase-free DNase at room temperature for $30 \mathrm{~min}$ utes (QIAGEN Inc.) and was quantified spectophotometrically. Ten nanograms of DNase-treated RNA was used to synthesize cDNA by random-primer reverse transcriptase (Perkin-Elmer Corp., Norwalk, Connecticut, USA). The cDNA was amplified using two specific primers for the vIL-10 gene: $5^{\prime}$-CATCATGTATGCTTCTAT GCAGTT-3' (upstream) and 5'-CCTGGCTCAGCACTGCT ATGCTGC-3' (downstream). Thirty PCR cycles using a thermocycler (Pekin-Elmer Corp.) yielded a 540-bp fragment. GAPDH was amplified from the same cDNA in a separate reaction using the same PCR cycle settings.

Conditioning of supernatants with ECs. ECs were prepared from WT FVB mice and vIL-10Tg mice (20). ECs were cultured in RPMI 1640 supplemented with $10 \%$ heat-inactivated FCS, 100 units/ml penicillin, 100 $\mathrm{mg} / \mathrm{ml}$ streptomycin, $0.1 \mathrm{mM}$ essential and nonessential amino acids, $2 \mathrm{mM}$ L-glutamine, $1 \mathrm{mM}$ sodium pyruvate, and 0.01 M HEPES buffer ("complete medium"). For conditioning of supernatants, ECs were cultured at a concentration of $1.5 \times 10^{6} \mathrm{cells} / \mathrm{ml}$, and supernatants were collected 48 hours after culture.

Preparation of serum. Blood was obtained from lethally anesthetized animals and allowed to clot at $4^{\circ} \mathrm{C}$. Serum was collected after centrifugation at $1,000 \mathrm{~g}$ for 5 minutes.

Quantitation of $v$ IL-10 by ELISA. VIL-10 levels in mouse serum and supernatant from fresh EC cultures were measured using a sandwich ELISA with an anti-vIL-10 $\mathrm{mAb}$ (Pharmingen, San Diego, California, USA). This antibody has significant cross-reactivity with human IL-10 and, in our experiments, appeared to have some cross-reactivity with murine IL-10 also. Briefly, microtiter plates were coated with $50 \mu \mathrm{l}$ purified anti-vIL-10 mAb's $(4 \mu \mathrm{g} / \mathrm{ml})$ overnight at $4^{\circ} \mathrm{C}$, then washed three times with PBS containing 0.05\% Tween-20 and blocked overnight at $4{ }^{\circ} \mathrm{C}$ with $4 \% \mathrm{BSA}$ in PBS. After washing of the plate four times, $100 \mu \mathrm{l}$ of standards and test samples were incubated in triplicate for 4 hours at room temperature. Plates were then washed, and $100 \mu \mathrm{l}$ of biotinylated rat anti-vIL-10 detection antibody was added at a final concentration of $2 \mu \mathrm{g} / \mathrm{ml}$ followed by incubation for 1 hour at room temperature. After washing with PBS, wells were further incubated with a 1:2,000 dilution of avidin-HRP (Vector Laboratories Inc., Burlingame, California, USA) for 30 minutes, followed by extensive washing. Finally, $100 \mu \mathrm{l} /$ well of ABTS substrate was added $\left[0.03 \%\right.$ of $2,2^{\prime}$-azino-bis-(3-ethylbenzothiazoline-6-sulfonic acid) (Sigma-Aldrich, St. Louis, Missouri, USA) in $0.1 \mathrm{M}$ anhydrous citric acid (Fisher Scientific Co., Pittsburgh, Pennsylvania, USA) dissolved in $\mathrm{dd}_{2} \mathrm{O}$ at $\mathrm{pH} 4.35$, with $100 \mu \mathrm{l}$ of $3 \% \mathrm{H}_{2} \mathrm{O}_{2}$ added to $11 \mathrm{ml}$ of this solution prior to use], and incubation was performed at room temperature for $30 \mathrm{~min}$ utes. The reaction was stopped by the addition of $50 \mu \mathrm{l}$ of $0.5 \mathrm{~N} \mathrm{H}_{2} \mathrm{SO}_{4}$ to each well. Absorbency was read at 405 $n m$ with a VERSAmax microplate reader (Molecular Devices Corp., Menlo Park, California, USA).

Immunohistochemical staining for $I-A$. Truncal skin was removed from FVB WT and vIL-10Tg mice, and frozen sections were prepared according to a standard protocol. Slides were defrosted, washed twice with PBS, and then incubated in PBS buffer containing 1\% BSA, NP-40, and peroxidase for 2 minutes, followed by incubation in PBS buffer containing 1\% BSA and 0.1\% NP-40 for 10 minutes. One hundred microliters of biotin-conjugated mouse anti-mouse I- $\mathrm{A}^{\mathrm{q}} \mathrm{mAb}$ (Pharmingen) at 1:100 dilution was placed onto the slides, followed by incubation for 1 hour at room temperature. An irrelevant biotin-conjugated mouse $\mathrm{mAb}$ was used as an isotype control. After washing of the slides with PBS five times, they were incubated with streptavidin-HRP at 1:500 dilution in $50 \mathrm{mM}$ Tris buffer ( $\mathrm{pH}$ 7.4) at room temperature for 30 minutes. After washing with Tris buffer three times, $400 \mu \mathrm{l}$ of $3,3^{\prime}$-diaminobenzidine solution 
was added for a 5 -minute incubation. After the color developed, the slides were rinsed with water three times and counterstained with hematoxylin. I- $\mathrm{A}^{\mathrm{q}^{+}}$cells were counted in a coded fashion using an ocular grid and are expressed as cells $/ \mathrm{mm}^{2}$ (mean \pm SEM; $\left.n=4\right)$.

Flow cytometry. ECs were prepared from WT FVB mice and vIL-10Tg mice. To determine the I-A ${ }^{9}$-positivity of LCs, purified anti-mouse I-A and isotype-matched control antibody (Pharmingen) were incubated with ECs on ice for 30 minutes, followed by incubation with FITC-conjugated anti-mouse IgG2a. For examination of CD80 and CD86 expression, double staining was achieved by incubation of phycoerythrin-conjugated (PE-conjugated) anti-mouse CD80 or anti-mouse CD86 with ECs after staining with FITC-I-Aq. Then FACScan analysis was performed (Becton Dickinson Immunocytometry Systems, San Jose, California, USA).

$D T H$ assay. Spleens were harvested from naive allogeneic mice $\left(\mathrm{CAF}_{1}, \mathrm{H}-2^{\mathrm{a} / \mathrm{d}}\right)$, and a suspension of cells was prepared by mechanical disaggregation. Cells were filtered through nylon gauze, and erythrocytes were lysed with $0.15 \mathrm{M}$ ammonium chloride. Splenocytes were then washed three times with PBS. Splenocytes $\left(1.5 \times 10^{7}\right.$ in $0.1 \mathrm{ml}$ ) were injected subcutaneously into each flank of each vIL-10Tg or WT mouse. Six days after immunization, these mice were challenged by subcutaneous injection of the left hind footpad with $2 \times 10^{8} \mathrm{CAF}_{1}$ splenocytes in $50 \mu \mathrm{l}$ PBS. Two groups of naive mice (vIL-10Tg and WT) were challenged in the same way and served as control groups. Footpad swelling was measured after 24 hours using an engineer's micrometer (Mitutoyo Corp., Tokyo, Japan), and swelling responses were calculated as the thickness of the challenged left footpad minus the thickness of the untreated right footpad.

CHS assay. vIL-10Tg and WT mice were immunized with either $25 \mu \mathrm{l}$ of $0.5 \%$ dinitrofluorobenzene (DNFB) or $5 \%$ oxazolone (Sigma-Aldrich) in acetone/sesame oil (4:1) on the clipped lower dorsum. Six days later, these mice and groups of nonimmunized mice (negative control) were challenged by painting of $5 \mu \mathrm{l}$ of either $0.2 \%$ DNFB or $1 \%$ oxazolone onto each side of each ear after measurement of base-line ear thickness with an engineer's micrometer (Mitutoyo Corp.). Ear thickness was measured again at 24 and 48 hours after challenge, and ear swelling was assessed as a measure of CHS.

Mixed EC-lymphocyte reaction. Two hundred thousand nylon wool-enriched allogeneic splenic $\mathrm{T}$ cells $\left(\mathrm{C}_{3} \mathrm{H} /\right.$ heJ, $\left.\mathrm{H}-2^{\mathrm{k}}\right)$ were cocultured with various numbers of freshly prepared $x$-irradiated (2.9 Gy) ECs from vIL-10Tg and WT mice (H-2q). The cells were cocultured in RPMI (Cellgro; Mediatech Inc., Herndon, Virginia, USA) supplemented with $10 \%$ FCS, $10 \mathrm{mM}$ HEPES buffer, $5 \mu \mathrm{g} / \mathrm{ml}$ indomethacin (SigmaAldrich), and $2.5 \times 10^{-5} \mathrm{M} 2$-mercaptoethanol at $37^{\circ} \mathrm{C}$ in round-bottomed 96 -well plates. Six days after coculture, all wells were pulsed with $1 \mu \mathrm{Ci}$ of ${ }^{3} \mathrm{H}$-thymidine for an additional 18 hours of culture. Then cells were harvested, and ${ }^{3} \mathrm{H}$-thymidine incorporation was measured by $\beta$-scintillation counting.
Mixed lymphocyte reaction. Two thousand allogeneic splenocytes $\left(\mathrm{BALB} / \mathrm{c}, \mathrm{H}-2^{\mathrm{d}}\right)$ were cocultured with various numbers of $\mathrm{x}$-irradiated (2.9 Gy) splenocytes from vIL-10Tg mice or WT mice (H-29). The cells were cocultured in RPMI (Cellgro; Mediatech Inc.) supplemented with $10 \%$ FCS, $10 \mathrm{mM}$ HEPES buffer, $5 \mu \mathrm{g} / \mathrm{ml}$ indomethacin (Sigma-Aldrich), and $2.5 \times 10^{-5} \mathrm{M} 2$-mercaptoethanol at $37^{\circ} \mathrm{C}$ in round-bottomed 96 -well plates. Six days after coculture, all wells were pulsed with $1 \mu \mathrm{Ci}$ of ${ }^{3} \mathrm{H}$-thymidine for an additional 18 hours of culture. Then cells were harvested, and ${ }^{3} \mathrm{H}$-thymidine incorporation was measured by $\beta$-scintillation counting.

LC migration assay. Groups of vIL-10Tg and WT mice were painted on the clipped thorax and abdomen with $400 \mu \mathrm{l}$ of $0.5 \%$ FITC in $1: 1$ acetone/dibutyl phthalate. Mice were sacrificed 24 hours later, and their inguinal and axillary lymph nodes were excised and pooled. The lymph nodes were gently disrupted to yield a suspension of cells and filtered through 70- $\mu \mathrm{M}$ nylon mesh. To enrich for DCs, lymph node cells were adjusted to $5 \times 10^{6}$ cells $/ \mathrm{ml}$, and then $6 \mathrm{ml}$ of the cell suspension was gently underlaid with $3 \mathrm{ml}$ of $14.5 \%$ metrizamide (Spectrum Quality Products Inc., New Brunswick, New Jersey, USA), followed by centrifugation at $300 \mathrm{~g}$ for 20 minutes. The low-buoyant-density population of cells (DCs) at the interface were collected, washed twice with RPMI containing 10\% FCS, and then resuspended in PBS containing $0.02 \%$ sodium azide and $1 \%$ FCS. Immunostaining was performed by incubation of DCs with purified anti-mouse I-Aq (Pharmingen) for 30 minutes on ice, followed by washing and staining with PE-labeled antimouse IgG2a (Pharmingen) and analysis by FACScan (Beckman Coulter Inc., Hialeah, Florida, USA).

EDTA separation and ATPase staining of LCs in the mouse epidermis. ATPase staining of LCs was performed by the method of Baker and Habowsky (21). Briefly, hair was removed from skin specimens by plucking, and subcutaneous fat and panniculus carnosus were removed by blunt dissection. Skin samples $4 \mathrm{~mm}^{2}$ in area were placed dermis-side-up onto the adhesive surface of white tape. Specimens were then transferred dermis-side-down to 20 $\mathrm{mM} \mathrm{Na}_{2}$-EDTA in PBS containing 1\% phenol red and were incubated for 3 hours. Epidermal sheets were separated from the dermis with forceps and rinsed in PBS supplemented with $0.25 \mathrm{ml}$ of $5 \% \mathrm{MgSO}_{4}$. EDTA-separated epidermis was stained for ATPase activity using the following buffers: (a) Trismal buffer (6.84\% sucrose), $\mathrm{pH}$ 7.3 , for 20 minutes (three changes) at $4^{\circ} \mathrm{C}$; (b) cacodylate-

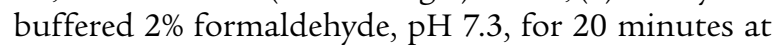
$4^{\circ} \mathrm{C}$; (c) Trismal buffer (6.84\% sucrose), $\mathrm{pH} 7.3$, for 20 minutes (two changes) at $4^{\circ} \mathrm{C}$; (d) ATP-Pb staining solution containing $10 \mathrm{mg}$ ATP- $\mathrm{Na}_{2}$ (vanadium-free; SigmaAldrich), $5 \mathrm{ml} 5 \% \mathrm{MgSO}_{4}, 3 \mathrm{ml} 2 \% \mathrm{~Pb}\left(\mathrm{NO}_{3}\right)_{2}$, and $42 \mathrm{ml}$ Trismal buffer (8.55\% sucrose), $\mathrm{pH} 7.3$, for 15 minutes at $37^{\circ} \mathrm{C}$; (e) Trismal buffer (6.84\% sucrose), $\mathrm{pH} 7.3$, for 20 minutes (two changes) at $4^{\circ} \mathrm{C}$; (f) $1 \%$ solution of $22.3 \%$ ammonium sulfide for 20 minutes at $4^{\circ} \mathrm{C}$; and (g) Trismal buffer (6.84\% sucrose), $\mathrm{pH} 7.3$, for 10 minutes (two changes) at $4^{\circ} \mathrm{C}$. After staining, epidermal sheets were 
mounted dermis-side-up in glycerin/PBS (9:1) and sealed with paraffin. ATPase $e^{+}$cells were counted using an ocular grid and are expressed as cells $/ \mathrm{mm}^{2}$ (mean \pm SEM; $n=4$ for transgenic mice and $n=3$ for WT mice).

UVB radiation-induced carcinogenesis. WT and VIL-10Tg mice (20 mice per group) were exposed to $2.16 \mathrm{~kJ} / \mathrm{m}^{2}$ of UVB radiation from FS40 sunlamps three times per week for 27 weeks. Mice were examined weekly for the development of skin tumors. Tumors that enlarged progressively were scored as malignant with onset on the date they were first noted.

NK cell activity. Splenocytes (effector cells) were isolated from WT mice and vIL-10Tg mice by mechanical dissociation and lysing of erythrocytes. YAC-1 cells (American Type Culture Collection, Rockville, Maryland, USA), a mouse lymphoma line sensitive to the cytotoxic activity of NK cells, were used as targets. YAC-1 cells were maintained in RPMI (Cellgro; Mediatech Inc.) supplemented with $10 \mathrm{mM}$ HEPES, $1 \mathrm{mM}$ sodium pyruvate, $4.5 \mathrm{~g} / \mathrm{lglu}-$ cose, $1.5 \mathrm{~g} / \mathrm{l}$ sodium bicarbonate, and 10\% FCS (Gemini BioProducts, Woodland, California, USA). Lactic dehydrogenase (LDH) release assay was used for measuring NK cell activity. Splenocytes and YAC-1 cells were washed three times in medium 199 (GIBCO BRL; Life Technologies Inc., Grand Island, New York, USA) containing 25 mM HEPES buffer, $50 \mu \mathrm{g} / \mathrm{ml}$ gentamicin, and $2 \%$ BSA, without phenol red (199-albumin medium). To remove the $\mathrm{LDH}$ that is present in FBS, YAC- 1 cells were washed three times prior to use and diluted to $2 \times 10^{4}, 1 \times 10^{4}$, $5 \times 10^{5}, 2.5 \times 10^{5}$, and $1.25 \times 10^{5}$ viable cells $/ \mathrm{ml}$; splenocytes were diluted to a concentration of $3 \times 10^{7} \mathrm{cells} / \mathrm{ml}$. One-tenth milliliter of target cells (YAC-1) and one-tenth milliliter of effector cells (splenocytes) were added to each well of round-bottomed 96 -well microtiter plates in triplicate. Then the plates were centrifuged at $45 \mathrm{~g}$ for $5 \mathrm{~min}$ utes and incubated at $37^{\circ} \mathrm{C}$ in $5 \% \mathrm{CO}_{2}$ for 4 hours. After 4 hours of incubation, $0.05 \mathrm{ml}$ of cold 199-albumin medium was added to each well, the plate was centrifuged at $250 \mathrm{~g}$ for 5 minutes, and $0.1-\mathrm{ml}$ aliquots of supernatant were transferred to the corresponding wells of an optically clear flat-bottomed microtiter plate. Next, $0.1 \mathrm{ml}$ of LDH substrate $\left[5.4 \times 10^{-2} \mathrm{M} \mathrm{L}(+)\right.$ lactate, $6.6 \times 10^{-4} \mathrm{M}$ 2-p-iodophenyl-3-p-nitrophenyl tetrazolium chloride, $2.8 \times 10^{-4} \mathrm{M}$ phenazine methosulfate, and $1.3 \times 10^{-3} \mathrm{M}$ NAD in $0.2 \mathrm{M}$ Tris buffer, $\mathrm{pH}$ 8.2] was added to each well. A microtiter plate reader (Molecular Devices Corp., Sunnyvale, California, USA) was used to monitor the absorbency at $490 \mathrm{~nm}$. Percentage cytotoxicity was calculated using the formula

\section{Equation 1}

$$
\% C=\frac{E-S-e}{M-S} \times 100
$$

where $E$ is the experimental release of LDH activity from target cells (YAC-1) incubated in the presence of effector cells (lymphocytes), $S$ is the spontaneous release of LDH activity from target cells (YAC-1) incubated in the absence of lymphocytes, $e$ is the spontaneous release of LDH activity from effector cells (lymphocytes), and $M$ is the maximal release of LDH activity determined by lysing of variable concentrations of target cells with $1 \%$ Triton X-100.

Statistical evaluation. The significance of differences between groups was assessed by Student's two-tailed $t$ test for unpaired samples, except for the tumor-growth experiment, in which Kaplan-Meier analysis was employed with the log-rank test, and the analysis of NK cell activity, in which the repeated-measures ANOVA test was used. A $P$ value of $\leq 0.05$ was considered significant.

\section{Results}

ECs from vIL-10Tg mice express $m R N A$ for vIL-10 and secrete $v I L-10$ protein. ECs were prepared from vIL-10Tg and WT FVB mice, total cellular RNA was isolated, and RT-PCR was performed using specific vIL-10 primers. As shown in Figure 1a, no expression of vIL-10 mRNA could be detected in WT mice; mice heterozygous for vIL-10

\section{Figure 1}

ECs from vIL-10Tg mice express mRNA for vIL-10 and secrete vIL-10 protein. (a) RT-PCR. Lane 1, DNA standard; lane 2, WT mice; lane 3, heterozygote transgenic mouse; lanes 4-8, homozygote transgenic mice; lane 9, RNA/PCR without RT. (b) vIL-10 in supernatants from fresh EC cultures and serum was measured by ELISA. (c) vIL-10 could be detected in serum from vIL-10Tg mice. $n=5$, both groups. Error bars represent $\pm \mathrm{SE}$. (b) $P<0.0005$, vlL-10Tg vs. WT; (c) $P=0.0005$, vlL-10Tg vs. WT.
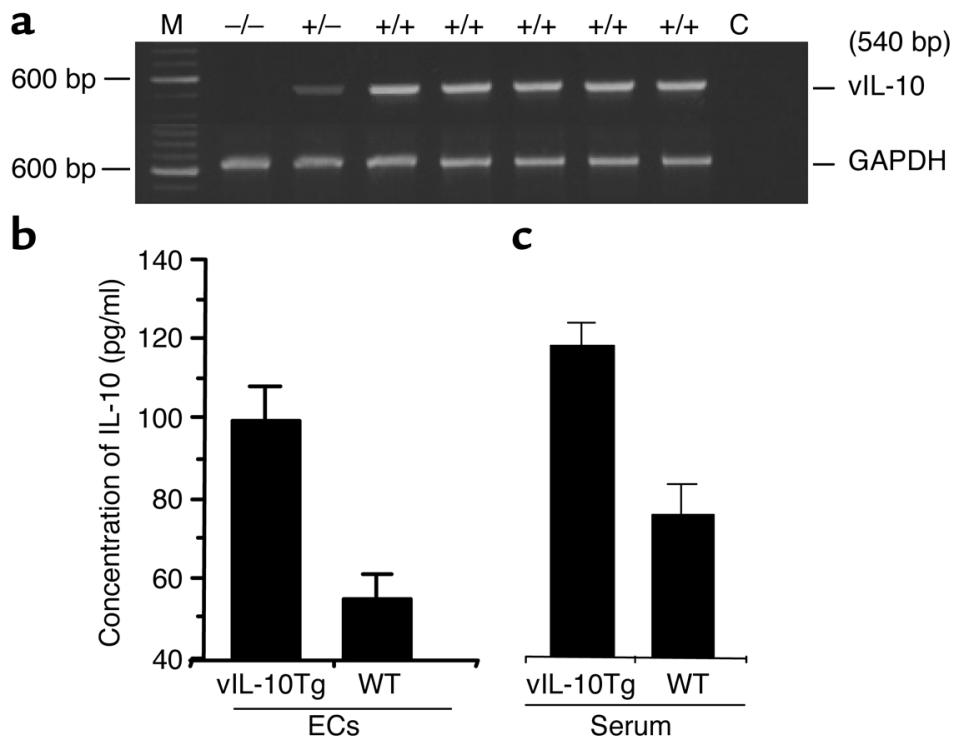
showed expression of a low level of vIL-10 mRNA, while homozygotes expressed high levels of vIL-10 mRNA.

To determine whether vIL-10Tg mice produce vIL-10 protein, we analyzed serum vIL-10 levels and EC-conditioned supernatants from WT and transgenic mice using a sandwich ELISA. The assay cross-reacted somewhat with murine IL-10. Nonetheless, the production of vIL-10 is demonstrated by the finding that the ELISA detected significantly more IL-10 in the supernatants conditioned by ECs from transgenic mice compared with WT mice (Figure 1b). Similarly, significantly increased levels of IL-10 were detected in the serum of transgenic mice compared with WT mice (Figure 1c).

The number of I- $A^{+}$cells is reduced in the epidermis and dermis of $v I L-10 T g$ mice and expression of I-A is suppressed, but a normal number of ATPase cells is present in vIL-10Tg mice. ECs were prepared from WT FVB mice and vIL-10Tg mice, stained with $\mathrm{I}-\mathrm{A}^{\mathrm{q}} \mathrm{mAb}$, and then analyzed by FACS. As shown in Figure 2a, a significant decrease in the proportion of cells that expressed abundant I-A ${ }^{\mathrm{q}}$ was observed in vIL-10Tg mice $(2.01 \%)$ compared with WT mice (4.49\%). Figure $2 \mathrm{~b}$ shows the average percentage of $\mathrm{I}-\mathrm{A}^{+}$cells in a group of ten mice of each type. The intensity of expression of I-A was also decreased in ECs from the transgenic mice (Figure 2c).

Immunohistochemical staining of frozen sections of skin for I-Aq showed a clear decrease in the number of I-A $\mathrm{A}^{+}$cells in the dermis of vIL-10Tg mice (Figure 2d). $\mathrm{I}^{-} \mathrm{A}^{+}$cells were enumerated in a coded fashion by microscopy using an ocular grid; the number of cells $/ \mathrm{mm}^{2}$ in WT specimens was $142.9 \pm 27.1$ (SEM), compared with $46.2 \pm 6.8$ in vIL-10Tg specimens $(P=0.018, n=4$ for each group).

To determine whether overexpression of the vIL-10 gene altered the density of epidermal LCs or only reduced I-A expression, epidermal sheets were obtained from vIL-10Tg mice and WT mice and stained for ATPase. The density of ATPase ${ }^{+}$cells found in the epidermis of vIL-10Tg mice $\left(1,205 \pm 86\right.$ [SEM] cells $\left./ \mathrm{mm}^{2}\right)$ was similar to that observed in WT mice $(1,203 \pm 99$ cells $/ \mathrm{mm}^{2}$ ) (Figure 2e).

The proportion of $\mathrm{I}^{-} \mathrm{A}^{+}$cells was not changed in the spleen according to FACS analysis.

Fewer numbers of hapten-bearing cells are detected in draining lymph nodes of vIL-10Tg mice, compared with WT controls, after hapten painting. Groups of vIL-10Tg mice and WT mice were skin-painted with FITC. Eighteen hours later, the draining lymph nodes were collected and suspensions of lymph node cells were prepared. A fraction enriched for DCs was obtained by separation on a metrizamide gradient and then immunolabeled with purified anti-I-Aq followed by staining with $\mathrm{PE}-$ conjugated anti-IgG2a (Figure 3a). The proportion of I- $\mathrm{A}^{+}$, FITC-bearing cells in the draining lymph nodes was lower in vIL-10Tg mice (18\%) than in WT mice (26.1\%). Figure 3 a shows data from a single transgenic and a single WT mouse. Figure $3 \mathrm{~b}$ shows the average number of I-A $A^{+}$FITC-bearing cells in a group of seven mice of each type. The difference observed was significant $(P=0.006)$.
Levels of expression of the costimulatory molecules CD80 and $C D 86$ are reduced in $I-A^{+} E C s$ from vIL-10Tg mice. ECs were prepared from vIL-10Tg and WT mice, doublestained with PE-labeled monoclonal anti-CD80 or -CD86 and FITC-labeled anti-I-A, and analyzed by FACS. The results showed significantly decreased expression of CD80 and CD86 in vIL-10Tg mice compared with WT animals after culture for 72 hours in vitro (Figure 4, $a$ and $b$ ).

ECs and splenocytes from vIL-10Tg mice have a decreased ability to stimulate allogeneic $T$ cell proliferation. As discussed above, ECs from transgenic mice secrete increased vIL-10 in vitro. To examine whether this vIL-10 affected the
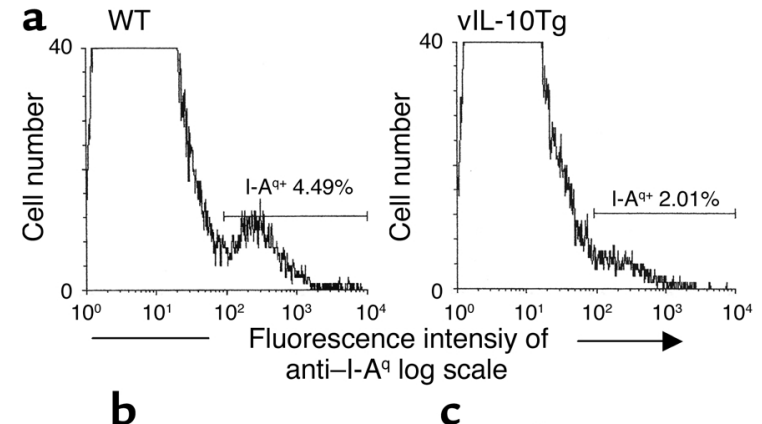

b
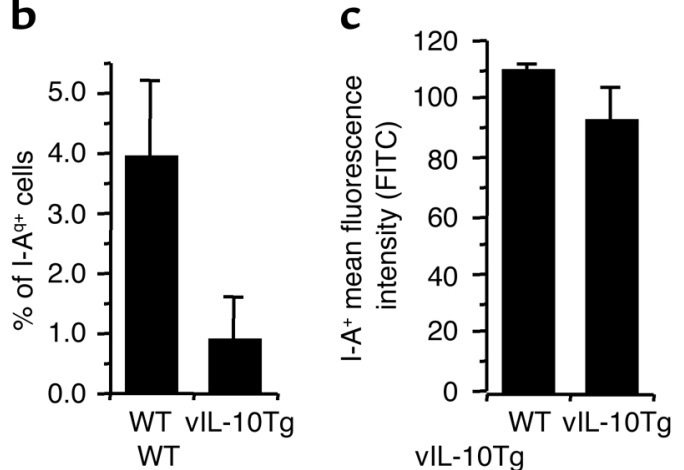

d
e
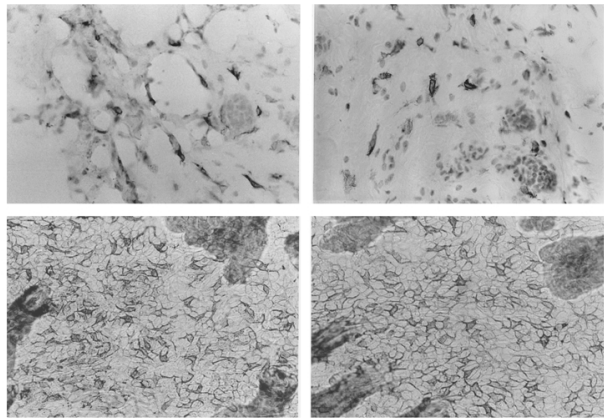

Figure 2

$\mathrm{I}-\mathrm{A}^{+}$expression is reduced in the epidermis and dermis of vIL-10Tg, although a normal proportion of ATPase ${ }^{+}$cells is present. (a) FACS analysis of $\mathrm{I}-\mathrm{A}^{+} \mathrm{EC}$ from a single transgenic and a single WT mouse. (b) Comparison of mean percentage of $\mathrm{I}^{-\mathrm{A}^{+}}$cells from groups of ten WT mice and ten vIL-10Tg mice. $P=0.0001$. (c) Mean intensity of I-Aq expression of vIL-10Tg mice and WT mice. $n=5$, both groups; $P=0.0124$. (d) Frozen sections of truncal skin from vlL-10Tg and WT mice were prepared, and staining for $\mathrm{I}-\mathrm{A}{ }^{q}$ was carried out. Decreased numbers of I-A 9 cells were seen in the dermis of vIL-10Tg mice. (e) Normal numbers of ATPase ${ }^{+}$cells are present in vIL-10Tg mice. Epidermal sheets were prepared from the dorsum of vIL-10Tg mice and WT mice and stained for ATPase activity. 
a

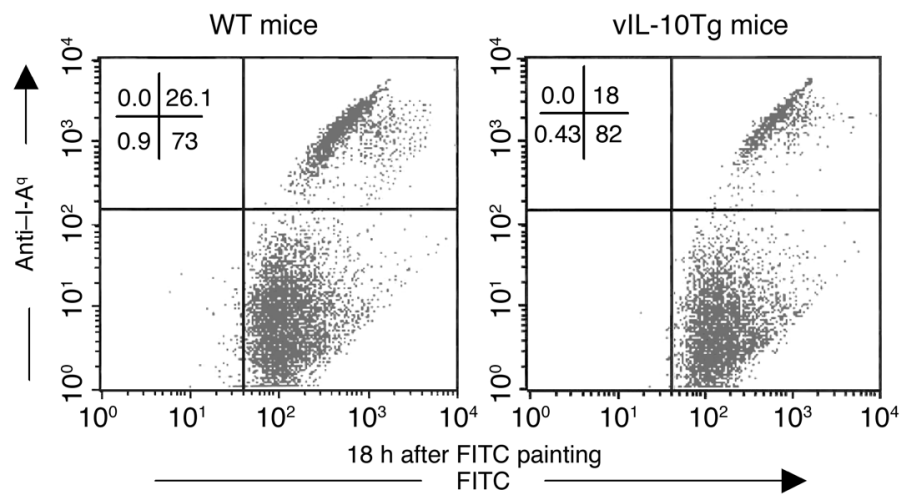

b

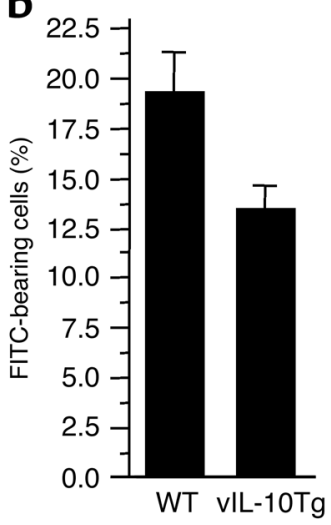

Figure 3

Skin painting with FITC yields fewer hapten-bearing I- $\mathrm{A}^{+}$cells in draining lymph nodes. VIL-10Tg and WT mice were painted on the thorax and abdomen with FITC, and 18 hours later, inguinal and axillary lymph nodes were collected. DCs were isolated, and two-color analysis for I-A 9 and FITC was performed. (a) The difference in frequency of FITC-bearing cells from a single vIL-10Tg mouse and a single WT mouse. (b) Comparison of mean frequency of hapten-bearing $\mathrm{I}-\mathrm{A}^{+}$cells from groups of seven VIL-10Tg mice and seven WT mice. Error bars represent \pm SEM. $P=0.006$, vlL-10Tg vs. WT.

ability of ECs to stimulate allogeneic $\mathrm{T}$ cell proliferation, we assessed the ability of ECs from transgenic and WT mice to present alloantigens to allogeneic T cells in the mixed EC-lymphocyte reaction. As shown in Figure 5a, ECs from vIL-10Tg mice had significantly reduced alloantigen-presenting ability compared with ECs from WT mice. We also assessed the ability of splenocytes from transgenic and WT mice to present alloantigens to $\mathrm{T}$ cells in the one-way mixed lymphocyte reaction. As shown in Figure 5b, splenocytes from vIL-10Tg mice had reduced alloantigen-presenting capability compared with lymphocytes from WT mice.

Expression of CD80 and CD86 by splenocytes was examined by FACS analysis. A statistically significant decrease in the proportion of CD80 and CD86 cells was observed in the transgenic mice (data not shown). However, the magnitude of the difference was extremely small.

DTH but not CHS is decreased in vIL-10Tg mice. To examine DTH, vIL-10Tg and WT mice were immunized with splenocytes from allogeneic mice and, 6 days later, were challenged in the left hind footpad. Negative control mice from each group were not immunized but were challenged. Twenty-four-hour footpad swelling was assessed as a measure of DTH. vIL-10Tg mice had a significantly decreased DTH response compared with WT control mice (Figure 6).

To examine CHS, vIL-10Tg and WT mice were sensitized by painting of DNFB onto the clipped dorsum and, 6 days later, were challenged by painting of DNFB onto the ears. Negative control mice from each group were not immunized but were challenged. Twenty-fourhour ear swelling was assessed as a measure of CHS. In contrast to the results seen in the DTH assay, ear swelling was not suppressed significantly in vIL-10Tg mice (data not shown).

vIL-10Tg mice develop fewer skin tumors in response to chronic UVB irradiation. VIL-10Tg and WT mice were chronically exposed to UVB radiation as described in
Methods and observed for skin cancer development. vIL-10Tg mice developed significantly fewer tumors than WT mice (Figure 7).

Splenic NK cells from vIL-10Tg mice demonstrate enhanced cytotoxic activity compared with splenic NK cells from WT mice. To examine the effect of vIL-10 production on NK cell activity in vivo, splenocytes from vIL-10 and WT mice were cocultured with YAC-1 cells for 4 hours. Splenocytes from vIL-10Tg mice exhibited enhanced NK cell activity compared with cells from WT mice $(P=0.01$; Figure 8$)$.

\section{Discussion}

IL-10 is of considerable interest because of its potent immunomodulatory properties and presumptive physiologic immunoregulation. Many studies have demonstrated a role for this cytokine in modulating
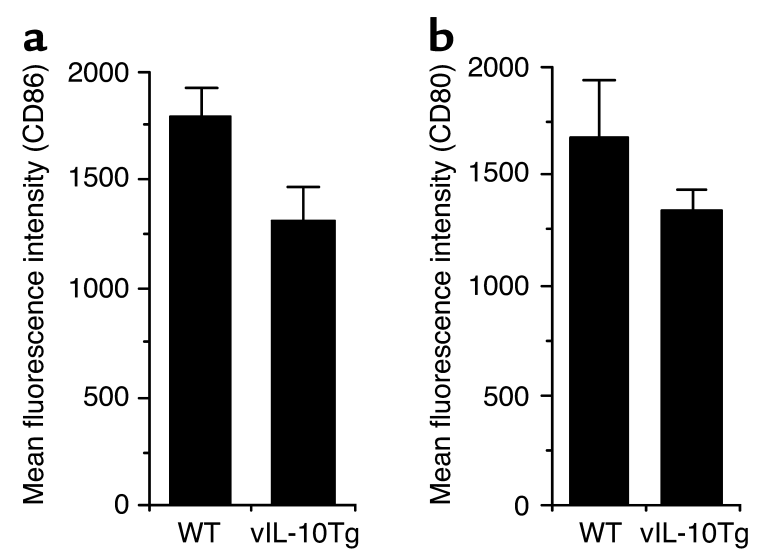

\section{Figure 4}

Cultured ECs from vlL-10Tg mice have decreased expression of CD80 and CD86. Fresh ECs were prepared from both vIL-10Tg and WT mice, cultured for 72 hours in vitro, and then stained with PE-labeled anti-CD80 or anti-CD86. The mean fluorescence intensity of CD80 (a) and CD86 (b) was assessed. $n=5$. (a) $P=0.0008$, vlL-10Tg vs. WT. (b) $P=0.03$, vlL-10Tg vs. WT. 

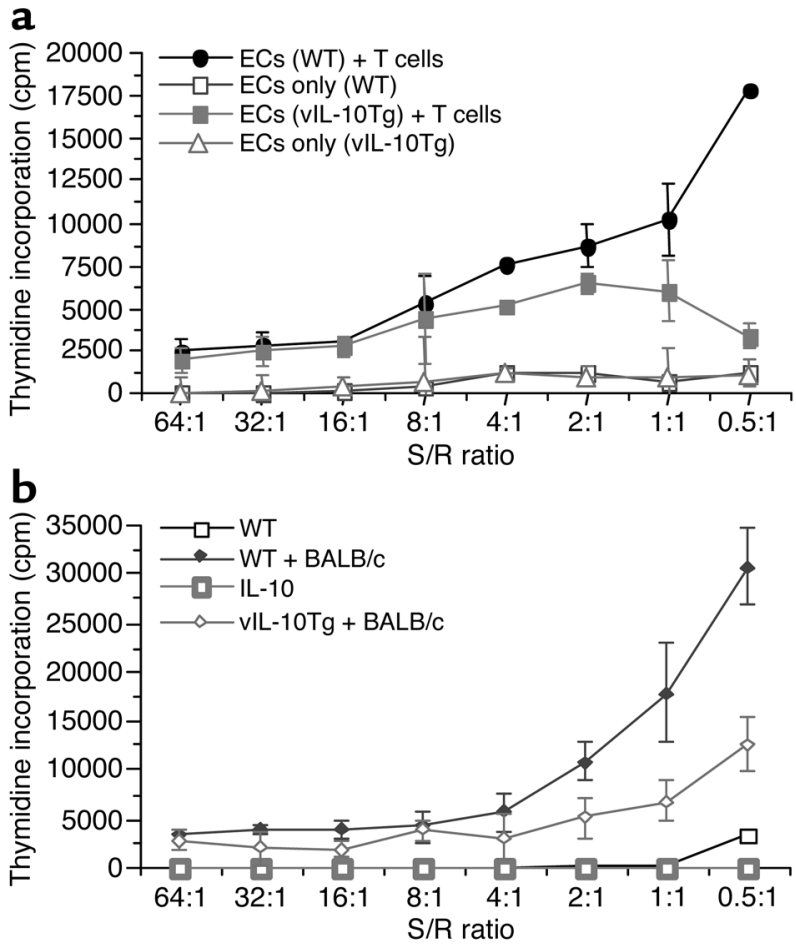

the balance of immunity versus tolerance to antigens encountered on or in the skin. IL-10 has also been found to play an important role in the immunosuppressive effects of exposure to UVB radiation (22-27). Therefore, we engineered transgenic mice that overexpress vIL-10 in the murine epidermis to further study the effects of this molecule in vivo.

Since IL-10 has effects on APCs, we examined the numbers of I-A $\mathrm{A}^{+}$cells in the epidermis and dermis of transgenic animals and compared them to the values found in WT mice. As discussed above, the proportion of I- $\mathrm{A}^{+}$ cells in the epidermis and the number of I- $\mathrm{A}^{+}$cells in the dermis were found to be significantly reduced in transgenic mice. Furthermore, $\mathrm{I}-\mathrm{A}^{+} \mathrm{ECs}$ from transgenic mice had a mean florescence intensity substantially below that observed in WT cells. This is reminiscent of a report that both cellular IL-10 and vIL-10 reduce MHC class II molecule expression on human monocytes (6). Interestingly, the density of ATPase ${ }^{+}$cells in epidermal sheets appeared to be normal in vIL-10Tg mice, suggesting that they may have a normal number of LCs but that expression of I-A is absent or severely reduced. Additionally, we

\section{Figure 6}

A decreased DTH response was observed in vIL-10Tg mice. WT mice (groups A and C) and vIL-10Tg mice (groups B and D) were examined. Groups of vIL-10Tg and WT mice were injected subcutaneously with splenocytes from naive allogeneic $\mathrm{CAF}_{1}$ mice (groups $\mathrm{C}$ and $\mathrm{D}$ ) or were not immunized (groups $A$ and $B$, negative control groups). Six days after immunization, all mice were challenged by subcutaneous injection of the left hind footpad with $\mathrm{CAF}_{1}$ splenocytes and 24-hour footpad swelling was assessed as a measure of DTH response. Error bars represent \pm SEM. $P=0.007, C$ vs. D; $P=\mathrm{NS}, A$ vs. B.

\section{Figure 5}

ECs and splenocytes from vlL-10Tg mice have decreased ability to stimulate allogeneic $T$ cell proliferation. (a) Various numbers of ECs (stimulators; S) were prepared from vIL-10Tg mice and WT mice. These cells were cocultured with $2 \times 10^{5}$ nylon wool-purified allogeneic $(B A L B / c)$ T cells (responders; R). Control ECs and T cells were cultured alone. $T$ cell proliferation was measured by ${ }^{3} \mathrm{H}$-thymidine uptake. Data are expressed as the mean cpm of triplicate cultures \pm SEM. (b) Various numbers of splenocytes (stimulators; S) were prepared from vIL-10Tg mice and WT mice and $x$-irradiated. These cells were cocultured with $2 \times 10^{5}$ allogeneic (BALB/c) splenocytes (responders; R). Stimulators and responders were cultured alone as controls. $T$ cell proliferation was measured by ${ }^{3} \mathrm{H}$-thymidine uptake. Data are expressed as the mean cpm of triplicate cultures \pm SEM.

found that expression of the costimulatory molecules CD80 and CD86 was greatly reduced on LCs from transgenic mice compared with WT mice after culture in vitro. This is of particular interest since these molecules play a crucial role in the activity of APCs. CD80 and CD86 differentially activate Th1/Th2 developmental pathways (28), and these molecules are expressed by epidermal LCs after culture or activation $(29,30)$. IL-10 reduces the expression of costimulatory molecules by APCs, including macrophages and DCs $(29,31)$. With respect to LCs, IL-10 inhibits the expression of both CD80 and CD86 $(30,32)$. In this regard, it is of interest that our experiments found that ECs from vIL-10Tg mice had a substantially decreased ability to stimulate allogeneic $\mathrm{T}$ cell proliferation in the mixed EC-lymphocyte reaction compared with ECs from WT control animals. There have been conflicting reports on the ability of IL-10 treatment of stimulator cells to inhibit this assay $(5,33)$; however, in this transgenic system a suppressed ability to stimulate was seen consistently. Our observation that DTH was reduced in vIL-10Tg mice but that CHS responses were normal is also of considerable interest. These findings support earlier studies suggesting that IL-10 mediates the suppressive effects of UVB radiation on induction of DTH, while TNF- $\alpha$ mediates the UVB radiation-induced suppression of CHS (27). Indeed, in gene-targeted, IL-10-deficient mice, UVB

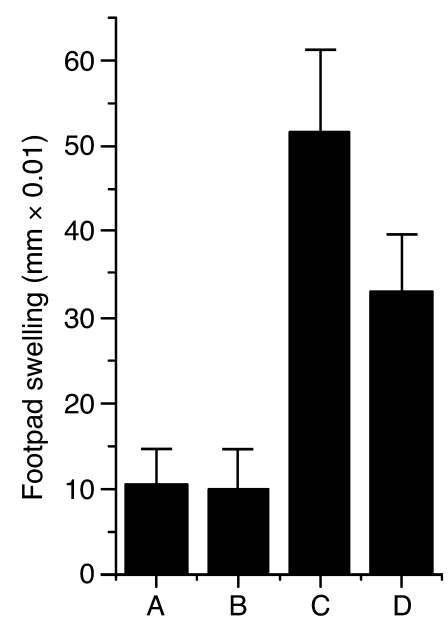




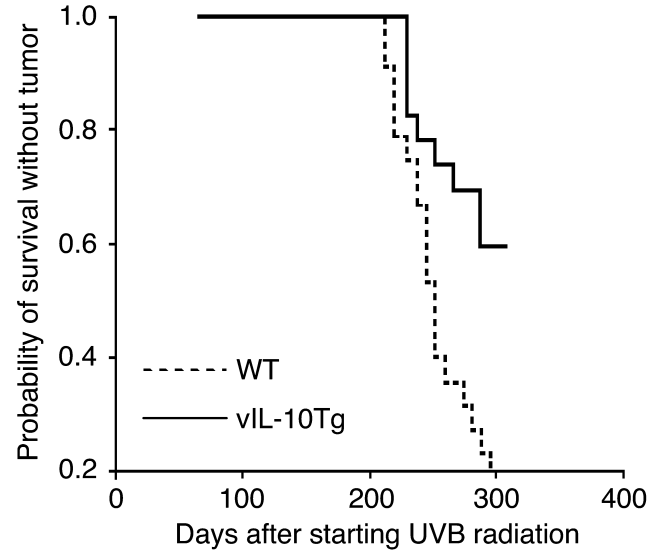

\section{Figure 7}

VIL-10Tg mice develop fewer skin tumors in response to chronic UVB irradiation. Groups of WT and VIL-10Tg mice were exposed to 2.16 $\mathrm{kJ} / \mathrm{m}^{2}$ of UVB irradiation from FS40 sunlamps three times per week for 27 weeks. Significantly fewer tumors developed over time in vlL-10Tg mice than in WT control mice ( $P<0.05 ; n=20$, both groups).

radiation inhibits the induction of CHS but has no effect on induction of DTH (22). Also, intraperitoneal administration of murine IL-10 suppresses the induction of DTH but not the induction of CHS (11). As a whole, these data support the concept that DTH and CHS are regulated by different mechanisms in vivo.

It has been hypothesized that one of the mechanisms by which UV radiation induces skin cancers is suppression of the normal immune response against neoplasms. If this is true, and IL-10 mediates suppression of a normal immune response against an incipient tumor, then vIL-10Tg mice may be more susceptible to cutaneous carcinogenesis. Since circumstantial evidence implicates IL-10 as a mediator of UVB radiation-induced immunosuppression, we hypothesized that vIL-10Tg mice would be more susceptible to UVB radiation-induced cutaneous carcinogenesis. However, our data indicated that chronically UVB-irradiated vIL-10Tg mice have a lower yield of tumors compared with WT animals. This was especially surprising given the report that a murine melanoma cell line engineered to secrete vIL-10 had enhanced tumor growth after inoculation into mice, while cells transduced with murine IL-10 had suppressed tumor growth (34). Similarly, myeloid DCs engineered to secrete vIL-10, when mixed with a sarcoma cell line and inoculated intradermally into syngeneic or allogenic mice, had enhanced tumor growth in both syngeneic and allogenic hosts. Transduction of myeloid DCs with murine IL-10, however, inhibited tumor development in this assay (35). On the other hand, introduction of vIL-10 genes into Burkitt lymphoma cells markedly reduced their ability to grow as subcutaneous tumors in SCID mice (36); the same study also demonstrated that vIL-10 reduced VEGF-165-induced neovascularization. The reasons for these discordant results are speculative at this time. The continual production of vIL-10 in the transgenic mice may induce receptor modulation or other downstream events that result in a different response from that seen in these other studies. Alternatively, the concentration of vIL-10 produced in vivo in these animals might be quite different from that seen in other models.

In order to explain these findings, we examined the angiogenic response, induction of CTL activity, and NK cell activity in vIL-10Tg and WT mice. To examine angiogenesis, we implanted 10 -mm-diameter polyvinyl acetate sponges subcutaneously into five vIL-10Tg and five WT mice. Twenty days later, the sponges were removed and vessel formation was assessed histologically. No significant difference in vessel formation was observed (data not shown). To examine specific CTL induction, vIL-10Tg and WT mice were immunized in vivo by subcutaneous injection with nucleated spleen cells from allogeneic $(\mathrm{BALB} / \mathrm{c})$ mice. Spleen cells from immunized mice were restimulated in vitro with spleen cells from $\mathrm{BALB} / \mathrm{c}$ mice for 5 days and then used in a 4-hour cytotoxicity assay with BALB/c concanavalin A lymphoblasts as targets. No significant difference in CTL activity between vIL-10Tg and WT mice was observed (data not shown). However, as described above, vIL-10Tg mice showed significantly enhanced NK cell activity compared with WT mice. This provides one possible explanation for our finding. Cellular IL-10 is known to augment NK cell activity and does so synergistically in combination with IL-18 $(37,38)$; however, when cellular IL-10 or vIL-10 was transfected into myeloid DCs that were used to generate NK effector cells in vitro, cellular IL-10 transfectants promoted increased NK cell activity while vIL-10 transfection did not yield a significant effect (35). Nonetheless, augmented NK cell activity was clearly evident in the

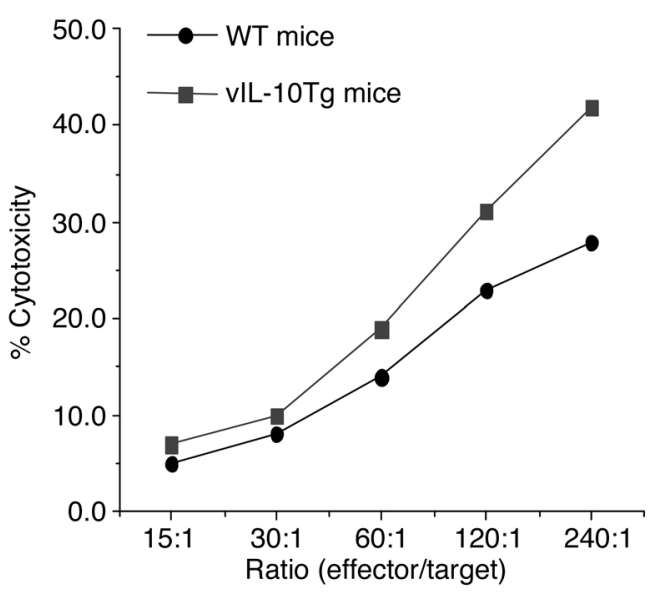

\section{Figure 8}

Splenocytes from vIL-10Tg mice demonstrate enhanced NK cell activity compared with splenocytes from WT mice. Varying numbers of splenocytes (effector cells) from vIL-10Tg and WT mice were incubated with YAC-1 cells (target cells) for 4 hours in round-bottomed 96-well plates. Cells from vIL-10Tg mice exhibited significantly greater NK cell activity than cells from WT mice $(P=0.01)$. 
vIL-10Tg mice. Overall, the data from the transgenic mice strongly indicate that vIL-10 may actually be inhibitory to the formation of skin tumors in response to UVB radiation.

In summary, our experiments have confirmed in a transgenic system that IL-10 inhibits a number of cutaneous immune functions. In combination with earlier observations by ourselves and others, these data support the concept that CHS and DTH are regulated differently and confirm the role of IL-10 in inhibiting DTH responses. Also, as a whole, these immunosuppressive effects support the idea that evolution has selected for expression of vIL-10 by certain viruses as part of a protective mechanism to inhibit immune-mediated attack by the host against virally infected cells. These results also suggest the possibility that IL-10 might have therapeutic applications in cutaneous immune-mediated diseases.

\section{Acknowledgments}

This work was supported by NIH grant R01 AR44240 (to R.D. Granstein), a grant from the Abercrombie Foundation, a grant from the Herbert and Ann Siegel Philanthropic Fund, a grant from the Edith C. Blum Foundation, a grant from Call on an Angel Inc., and contributions from Shiseido Co. Ltd. through the Massachusetts General Hospital/Harvard Cutaneous Biology Research Center and Wellman Laboratories of Photomedicine.

1. Taga, K., and Tosato, G. 1992. IL-10 inhibits human T cell proliferation and IL-2 production. J. Immunol. 148:1143-1148.

2. Del Prete, G., et al. 1993. Human IL-10 is produced by both type 1 helper (Th1) and type 2 helper (Th2) T cell clones and inhibits their antigenspecific proliferation and cytokine production. J. Immunol. 150:353-360.

3. Li, L., Elliott, J.F., and Mosmann, T.R. 1994. IL-10 inhibits cytokine production, vascular leakage, and swelling during $T$ helper 1 cell-induced delayed-type hypersensitivity. J. Immunol. 153:3967-3978.

4. Macatonia, S.E., Doherty, T.M., Knight, S.C., and O'Garra, A. 1993. Differential effect of IL-10 on dendritic cell-induced T cell proliferation and IFN-gamma production. J. Immunol. 150:3755-3765.

5. Enk, A.H., Angeloni, V.L., Udey, M.C., and Katz, S.I. 1993. Inhibition of Langerhans cell antigen-presenting function by IL-10. A role for IL-10 in induction of tolerance. J. Immunol. 151:2390-2398.

6. de Waal Malefyt, R., et al. 1991. Interleukin 10 (IL-10) and viral IL-10 strongly reduce antigen-specific human $\mathrm{T}$ cell proliferation by diminishing the antigen-presenting capacity of monocytes via downregulation of class II major histocompatibility complex expression. J. Exp. Med. 174:915-924.

7. Fiorentino, D.F., Zlotnik, A., Mosmann, T.R., Howard, M., and O'Garra, A. 1991. IL-10 inhibits cytokine production by activated macrophages. J. Immunol. 147:3815-3822.

8. Scott, P. 1993. IL-12: initiation cytokine for cell-mediated immunity. Science. 260:496-497.

9. Sypek, J.P., et al. 1993. Resolution of cutaneous leishmaniasis: interleukin 12 initiates a protective T helper type 1 immune response. J. Exp. Med. 177:1797-1802.

10. Chen, W.F., and Zlotnik, A. 1991. IL-10: a novel cytotoxic T cell differentiation factor. J. Immunol. 147:528-534.

11. Schwarz, A., et al. 1994. In vivo effects of interleukin-10 on contact hypersensitivity and delayed-type hypersensitivity reactions. J. Invest. Dermatol. 103:211-216.

12. Baer, R., et al. 1984. DNA sequence and expression of the B95-8 EpsteinBarr virus genome. Nature. 310:207-211.

13. Moore, K.W., et al. 1990. Homology of cytokine synthesis inhibitory factor (IL-10) to the Epstein-Barr virus gene BCRFI [erratum 1990, 250:494]. Science. 248:1230-1234.

14. Vieira, P., et al. 1991. Isolation and expression of human cytokine synthesis inhibitory factor cDNA clones: homology to Epstein-Barr virus open reading frame BCRFI. Proc. Natl. Acad. Sci. U. S. A. 88:1172-1176.

15. Fiorentino, D.F., Bond, M.W., and Mosmann, T.R. 1989. Two types of mouse T helper cell. IV. Th2 clones secrete a factor that inhibits cytokine production by Th1 clones. J. Exp. Med. 170:2081-2095.

16. Go, N.F., et al. 1990. Interleukin 10, a novel B cell stimulatory factor: unresponsiveness of X chromosome-linked immunodeficiency B cells. J. Exp. Med. 172:1625-1631.

17. MacNeil, I.A., Suda, T., Moore, K.W., Mosmann, T.R., and Zlotnik, A. 1990. IL-10, a novel growth cofactor for mature and immature $T$ cells. J. Immunol. 145:4167-4173.

18. Ma, Y., et al. 1998. Inhibition of collagen-induced arthritis in mice by viral IL-10 gene transfer. J. Immunol. 161:1516-1524.

19. Kim, K.N., et al. 2000. Viral IL-10 and soluble TNF receptor act synergistically to inhibit collagen-induced arthritis following adenovirusmediated gene transfer. J. Immunol. 164:1576-1581.

20. Grabbe, S., et al. 1991. Tumor antigen presentation by murine epidermal cells. J. Immunol. 146:3656-3661.

21. Baker, K.W., and Habowsky, J.E. 1983. EDTA separation and ATPase Langerhans cell staining in the mouse epidermis. J. Invest. Dermatol. 80:104-107.

22. Beissert, S., et al. 1996. Impaired immunosuppressive response to ultraviolet radiation in interleukin-10-deficient mice. J. Invest. Dermatol. 107:553-557.

23. Kurimoto, I., Kitazawa, T., and Streilein, J.W. 2000. Studies of delayed systemic effects of ultraviolet $B$ radiation (UVR) on the induction of contact hypersensitivity. II. Evidence that interleukin-10 from UVR-treated epidermis is the critical mediator. Immunology. 99:134-140.

24. Niizeki, H., and Streilein, J.W. 1997. Hapten-specific tolerance induced by acute, low-dose ultraviolet $B$ radiation of skin is mediated via interleukin-10. J. Invest. Dermatol. 109:25-30.

25. Shreedhar, V., Giese, T., Sung, V.W., and Ullrich, S.E. 1998. A cytokine cascade including prostaglandin E2, IL-4, and IL-10 is responsible for UV-induced systemic immune suppression. J. Immunol. 160:3783-3789.

26. Garssen, J., et al. 1999. UVB exposure-induced systemic modulation of Th1- and Th2-mediated immune responses. Immunology. 97:506-514.

27. Rivas, J.M., and Ullrich, S.E. 1994. The role of IL-4, IL-10, and TNF-alpha in the immune suppression induced by ultraviolet radiation. J. Leukoc. Biol. 56:769-775.

28. Yokozeki, H., et al. 1996. Functional CD86 (B7-2/B70) on cultured human Langerhans cells. J. Invest. Dermatol. 106:147-153.

29. Chang, C.H., Furue, M., and Tamaki, K. 1995. B7-1 expression of Langerhans cells is up-regulated by proinflammatory cytokines, and is downregulated by interferon-gamma or by interleukin-10. Eur. J. Immunol. 25:394-398.

30. Kawamura, T., and Furue, M. 1995. Comparative analysis of B7-1 and B7-2 expression in Langerhans cells: differential regulation by $\mathrm{T}$ helper type 1 and T helper type 2 cytokines. Eur. J. Immunol. 25:1913-1917.

31. Ding, L., Linsley, P.S., Huang, L.Y., Germain, R.N., and Shevach, E.M. 1993. IL-10 inhibits macrophage costimulatory activity by selectively inhibiting the up-regulation of B7 expression. J. Immunol. 151:1224-1234.

32. Powrie, F., Menon, S., and Coffman, R.L. 1993. Interleukin-4 and interleukin-10 synergize to inhibit cell-mediated immunity in vivo. Eur. J. Immunol. 23:3043-3049.

33. Peguet-Navarro, J., et al. 1994. Interleukin-10 inhibits the primary allogeneic $\mathrm{T}$ cell response to human epidermal Langerhans cells. Eur. J. Immunol. 24:884-891.

34. Suzuki, T., et al. 1995. Viral interleukin 10 (IL-10), the human herpes virus 4 cellular IL-10 homologue, induces local anergy to allogeneic and syngeneic tumors. J. Exp. Med. 182:477-486.

35. Takayama, T., Tahara, H., and Thomson, A.W. 2001. Differential effects of myeloid dendritic cells retrovirally transduced to express mammalian or viral interleukin-10 on cytotoxic T lymphocyte and natural killer cell functions and resistance to tumor growth. Transplantation. 71:1334-1340

36. Cervenak, L., et al. 2000. Abolished angiogenicity and tumorigenicity of Burkitt lymphoma by interleukin-10. Blood. 96:2568-2573.

37. Cai, G., Kastelein, R.A., and Hunter, C.A. 1999. IL-10 enhances NK cell proliferation, cytotoxicity, and production of IFN-gamma when combined with IL-18. Eur. J. Immunol. 29:2658-2665.

38. Parato, K.G., et al. 2002. Normalization of natural killer cell function and phenotype with effective anti-HIV therapy and the role of IL-10. AIDS. 16:1251-1256. 